\title{
Sobolev, Poincaré, and isoperimetric inequalities for subelliptic diffusion operators satisfying a generalized curvature dimension inequality
}

\author{
Fabrice Baudoin and Bumsik Kim
}

\begin{abstract}
By adapting some ideas of M. Ledoux ([12], [13] and [14]) to a sub-Riemannian framework we study Sobolev, Poincaré and isoperimetric inequalities associated to subelliptic diffusion operators that satisfy the generalized curvature dimension inequality that was introduced by F. Baudoin and N. Garofalo in [3]. Our results apply in particular on all CR Sasakian manifolds whose horizontal Webster-Tanaka-Ricci curvature is nonnegative, all Carnot groups with step two, and wide subclasses of principal bundles over Riemannian manifolds whose Ricci curvature is nonnegative.
\end{abstract}

\section{Introduction and framework}

In this paper, $\mathbb{M}$ will be a $C^{\infty}$ connected finite-dimensional manifold endowed with a smooth measure $\mu$ and a second-order diffusion operator $L$ on $\mathbb{M}$, locally subelliptic in the sense of [7] (see also [11]), satisfying $L 1=0$ and

$$
\int_{\mathbb{M}} f L g d \mu=\int_{\mathbb{M}} g L f d \mu, \quad \int_{\mathbb{M}} f L f d \mu \leq 0,
$$

for every $f, g \in C_{0}^{\infty}(\mathbb{M})$. We indicate by $\Gamma(f):=\Gamma(f, f)$ the carré du champ of $L$, that is the quadratic differential form defined by

$$
\Gamma(f, g)=\frac{1}{2}(L(f g)-f L g-g L f), \quad f, g \in C^{\infty}(\mathbb{M}) .
$$

There is an intrinsic distance associated to $L$ that can be defined via the notion of subunit curves (see [7]). An absolutely continuous curve $\gamma:[0, T] \rightarrow \mathbb{M}$ is said to be subunit for the operator $L$ if for every smooth function $f: \mathbb{M} \rightarrow \mathbb{R}$ we have

Mathematics Subject Classification (2010): Primary 58Jxx; Secondary 35A23, 35H20.

Keywords: Sobolev inequalities, isoperimetric inequality, Poincaré inequality, subelliptic operator. 
$\left|\frac{d}{d t} f(\gamma(t))\right| \leq \sqrt{(\Gamma f)(\gamma(t))}$. We then define the subunit length of $\gamma$ by $\ell_{s}(\gamma)=T$. Given $x, y \in \mathbb{M}$, we write

$$
S(x, y)=\{\gamma:[0, T] \rightarrow \mathbb{M} \mid \gamma \text { is subunit for } L, \gamma(0)=x, \gamma(T)=y\}
$$

In this paper we assume that $S(x, y)$ is not empty for every $x, y \in \mathbb{M}$. With such an assumption it is easy to verify that

$$
d(x, y)=\inf \left\{\ell_{s}(\gamma) \mid \gamma \in S(x, y)\right\}
$$

defines a true distance on $\mathbb{M}$. Furthermore, in this case, it is known that

$$
d(x, y)=\sup \left\{|f(x)-f(y)| \mid f \in C^{\infty}(\mathbb{M}),\|\Gamma(f)\|_{\infty} \leq 1\right\}, \quad x, y \in \mathbb{M} .
$$

Throughout this paper, we assume that the metric space $(\mathbb{M}, d)$ is complete.

In addition to the differential form (1.1), we assume that $\mathbb{M}$ is endowed with another smooth symmetric bilinear differential form, indicated by $\Gamma^{Z}$, satisfying, for $f, g \in C^{\infty}(\mathbb{M})$,

$$
\Gamma^{Z}(f g, h)=f \Gamma^{Z}(g, h)+g \Gamma^{Z}(f, h)
$$

and $\Gamma^{Z}(f)=\Gamma^{Z}(f, f) \geq 0$.

We make the following assumptions that will be in force throughout the paper:

(H.1) There exists an increasing sequence $h_{k} \in C_{0}^{\infty}(\mathbb{M})$ such that $h_{k} \nearrow 1$ on $\mathbb{M}$, and

$$
\left\|\Gamma\left(h_{k}\right)\right\|_{\infty}+\left\|\Gamma^{Z}\left(h_{k}\right)\right\|_{\infty} \rightarrow 0, \quad \text { as } k \rightarrow \infty .
$$

(H.2) For any $f \in C^{\infty}(\mathbb{M})$ one has

$$
\Gamma\left(f, \Gamma^{Z}(f)\right)=\Gamma^{Z}(f, \Gamma(f)) .
$$

(H.3) For every $t \geq 0, P_{t} 1=1$, and for every $f \in C_{0}^{\infty}(\mathbb{M})$ and $T \geq 0$, one has

$$
\sup _{t \in[0, T]}\left\|\Gamma\left(P_{t} f\right)\right\|_{\infty}+\left\|\Gamma^{Z}\left(P_{t} f\right)\right\|_{\infty}<+\infty
$$

where $P_{t}$ is the heat semigroup generated by $L$.

As has been proved in [3], the assumption (H.1) implies in particular that $L$ is essentially self-adjoint on $C_{0}^{\infty}(\mathbb{M})$. The assumption (H.2) is subtler and is crucial for the validity of most of the subsequent results: it is discussed in detail in [3] in several geometric examples. In the sub-Riemannian geometries covered by the present work (H.2) means that the torsion of the sub-Riemannian connection is vertical. Assumption (H.3) is necessary to rigorously justify the Bakry-Émery type arguments. In many examples it is a consequence of the generalized curvature dimension inequality below (see [3]). Other sufficient conditions ensuring that (H.3) is satisfied can be found in [17]. 
In addition to $\Gamma$ and $\Gamma^{Z}$ we need the following second order differential bilinear forms:

$$
\begin{aligned}
\Gamma_{2}(f, g) & =\frac{1}{2}[L \Gamma(f, g)-\Gamma(f, L g)-\Gamma(g, L f)] \\
\Gamma_{2}^{Z}(f, g) & =\frac{1}{2}\left[L \Gamma^{Z}(f, g)-\Gamma^{Z}(f, L g)-\Gamma^{Z}(g, L f)\right] .
\end{aligned}
$$

As for $\Gamma$ and $\Gamma^{Z}$, we will freely use the notations $\Gamma_{2}(f)=\Gamma_{2}(f, f)$ and $\Gamma_{2}^{Z}(f)=$ $\Gamma_{2}^{Z}(f, f)$.

The following curvature dimension condition was introduced in [3].

Definition 1.1. (See [3]) We say that $L$ satisfies the generalized curvature dimension inequality $C D\left(\rho_{1}, \rho_{2}, \kappa, d\right)$ if there exist constants $\rho_{1} \in \mathbb{R}, \rho_{2}>0, \kappa \geq 0$, and $0<d<\infty$ such that the inequality

$$
\Gamma_{2}(f)+\nu \Gamma_{2}^{Z}(f) \geq \frac{1}{d}(L f)^{2}+\left(\rho_{1}-\frac{\kappa}{\nu}\right) \Gamma(f)+\rho_{2} \Gamma^{Z}(f)
$$

holds for every $f \in C^{\infty}(\mathbb{M})$ and every $\nu>0$, where $\Gamma_{2}$ and $\Gamma_{2}^{Z}$ are defined by (1.4) and (1.5).

The motivation for this condition comes from the study of several examples coming from sub-Riemannian geometry where the generalized curvature dimension inequality turns out to be equivalent to lower bounds on intrinsic curvature tensors (see [3]). The parameter $\rho_{1}$ is of special importance. It is the curvature parameter. The condition $\rho_{1}=0$ means that the ambient space has nonnegative curvature whereas the condition $\rho_{1}>0$ means that it has positive curvature. In particular, in the latter case a Bonnet-Myers type theorem was proved in [3], implying that $\mathbb{M}$ must be compact. Let us mention that in [17], F. Y. Wang proposed an extension of the generalized curvature dimension inequality .

Our goal in the present work is to discuss Sobolev type embeddings, isoperimetric type results, and Poincaré inequalities by using the generalized curvature dimension inequality. Our methods will exploit and extend to the present subelliptic framework some clever and beautiful ideas due to M. Ledoux (see [12], [13] and [14] ) who used heat semigroup methods to study isoperimetric, Sobolev, and Poincaré inequalities. Our discussion will be based on the curvature parameter $\rho_{1}$.

In the case $\rho_{1}=0$, which is studied in Section 2, one of our main results is the following Besov-Sobolev embedding:

Theorem 1.2. Assume that $L$ satisfies the generalized curvature dimension inequality $\operatorname{CD}\left(0, \rho_{2}, \kappa, d\right)$. For every $1 \leq p<q<\infty$ and every $f \in W^{1, p}(\mathbb{M})$, we have

$$
\|f\|_{q} \leq C\|\sqrt{\Gamma(f)}\|_{p}^{\theta}\|f\|_{B_{\infty, \infty}^{\theta /(\theta-1)}}^{1-\theta}
$$

where $\theta=p / q$; where $C>0$ is a constant that only depends on $p, q, \rho_{2}, \kappa$ and $d$; and where $\|\cdot\|_{B_{\infty, \infty}^{\theta /(\theta-1)}}$ is the Besov norm which is introduced in (2.3). 
We then prove that this Besov-Sobolev embedding implies the following isoperimetric inequality:

Proposition 1.3. Assume that $L$ satisfies the generalized curvature dimension inequality $\operatorname{CD}\left(0, \rho_{2}, \kappa, d\right)$. Assume that there exists constants $C>0$ and $D>0$ such that for every $x \in \mathbb{M}$ and $R \geq 0, \mu(B(x, R)) \geq C R^{D}$. For any $1 \leq p, q, r<\infty$ with $1 / q=1 / p-r /(q D)$, there exists a constant $C^{\prime}>0$ such that for all $f \in$ $C_{0}^{\infty}(\mathbb{M})$, we have

$$
\|f\|_{q} \leq C^{\prime}\|\sqrt{\Gamma(f)}\|_{p}^{p / q}\|f\|_{r}^{1-p / q}
$$

and there exists a constant $C^{\prime \prime}>0$ such that, for every Caccioppoli set $E \subset \mathbb{M}$, one has

$$
\mu(E)^{(D-1) / D} \leq C^{\prime \prime} P(E)
$$

where $P(E)$ denotes the horizontal perimeter of $E$ in $\mathbb{M}$.

In the isoperimetric inequality (1.6) the constant $C^{\prime \prime}$ we obtain is not sharp but the exponent $(D-1) / D$ is correct as the example of the Heisenberg group, to which the result applies, shows. We can see that in the Euclidean case the optimal isoperimetric constant can be obtained from the semigroup method by using RieszSobolev rearrangement type inequalities (see [4] and [13]). However, so far, to the knowledge of the authors, the rearrangement inequality is not available in the Heisenberg group case. Since the celebrated note of Pansu [15], the problem of the optimal isoperimetric constant on the Heisenberg group is a long-standing open problem (see [5]).

In Section 3, we study the case where the curvature parameter $\rho_{1}$ is positive. In that case, as we stressed before, the manifold $\mathbb{M}$ must be compact and the measure $\mu$ must be finite. We obtain the following Poincaré inequality:

Proposition 1.4. Assume that $L$ satisfies the generalized curvature dimension inequality $\operatorname{CD}\left(\rho_{1}, \rho_{2}, \kappa, d\right)$ with $\rho_{1}>0$. Let $1 \leq p<\infty$. There exists $C=$ $C_{p}\left(\rho_{1}, \rho_{2}, \kappa, d\right)>0$ such that, for every $f \in C_{0}^{\infty}(\mathbb{M})$,

$$
\left\|f-f_{\mathbb{M}}\right\|_{p} \leq C\|\sqrt{\Gamma(f)}\|_{p}
$$

where $f_{\mathbb{M}}=\frac{1}{\mu(\mathbb{M})} \int_{\mathbb{M}} f d \mu$.

Interestingly, the constant $C$ we obtain is explicit and does not depend on $p$ for $1 \leq p<2$ or $2 \leq p<\infty$. Also $C$ does not depend on the dimension $d$ when $1 \leq p<2$.

The end of Section 3 is then devoted to the study of the isoperimetric constant introduced by Cheeger in [6] in a Riemannian framework and to the study of the first nonzero eigenvalue of $\mathbb{M}$. Concerning Cheeger's isoperimetric constant, we prove in particular the following lower bound. 
Proposition 1.5. Assume that $L$ satisfies the generalized curvature dimension inequality $\operatorname{CD}\left(\rho_{1}, \rho_{2}, \kappa, d\right)$ with $\rho_{1}>0$ and that $\mu(\mathbb{M})=1$. Define

$$
\iota=\inf \frac{P(E)}{\mu(E)},
$$

where the infimum runs over all Caccioppoli sets $E$ such that $\mu(E) \leq 1 / 2$. We have then

$$
\iota \geq \frac{1}{2} \sqrt{\frac{\rho_{1}}{2}} \frac{1}{1+2 \kappa / \rho_{2}} .
$$

Concerning the first eigenvalue we prove the following analogue of the celebrated Lichnerowicz' lower bound:

Proposition 1.6. Assume that $L$ satisfies the generalized curvature dimension inequality $\operatorname{CD}\left(\rho_{1}, \rho_{2}, \kappa, d\right)$ with $\rho_{1}>0$. The first nonzero eigenvalue $\lambda_{1}$ of $-L$ satisfies the estimate

$$
\lambda_{1} \geq \frac{\rho_{1} \rho_{2}}{\frac{d-1}{d} \rho_{2}+\kappa} .
$$

To conclude the introduction, let us now turn to the fundamental question of the examples to which the above results apply. We refer the reader to [3] for more details about most of the examples we discuss below.

Besides Laplace-Beltrami operators on complete Riemannian manifolds with Ricci curvature bounded from below, a wide class of examples is given by sub-Laplacians on sub-Riemannian manifolds with transverse symmetries. SubLaplacians on Sasakian manifolds form a special and interesting subclass that we quickly describe below. Let $\mathbb{M}$ be a complete strictly pseudoconvex CR Sasakian manifold with real dimension $2 n+1$. Let $\theta$ be a pseudo-Hermitian form on $\mathbb{M}$ with respect to which the Levi form is positive definite. The kernel of $\theta$ determines a horizontal bundle $\mathcal{H}$. Denote now by $T$ the Reeb vector field on $\mathbb{M}$, i.e., the characteristic direction of $\theta$. We recall that the $\mathrm{CR}$ manifold $(\mathbb{M}, \theta)$ is called Sasakian if $T$ is a sub-Riemannian Killing field. For instance the standard CR structures on the Heisenberg group $\mathbb{H}_{2 n+1}$ and the sphere $\mathbb{S}^{2 n+1}$ are Sasakian. On CR manifolds, there is a canonical subelliptic diffusion operator which is called the CR sub-Laplacian. It plays the same role in CR geometry as the Laplace-Beltrami operator does in Riemannian geometry. In this framework we have the following result that shows the relevance of the generalized curvature dimension inequality.

Proposition 1.7. [3] Let (M, $\theta$ ) be a complete CR Sasakian manifold with real dimension $2 n+1$. If for every $x \in \mathbb{M}$ the Tanaka-Webster Ricci tensor satisfies the bound

$$
\operatorname{Ric}_{x}(v, v) \geq \rho_{1}|v|^{2}
$$

for every horizontal vector $v \in \mathcal{H}_{x}$, then, for the $\mathrm{CR}$ sub-Laplacian of $\mathbb{M}$, the curvature dimension inequality $\operatorname{CD}\left(\rho_{1}, d / 4,1, d\right)$ holds with $d=2 n$ and $\Gamma^{Z}(f)=$ $(T f)^{2}$ and the hypotheses (H.1), (H.2), (H.3) are satisfied. 
In addition to sub-Laplacians on Heisenberg groups, more generally, the subLaplacian on any Carnot group of step 2 has been shown to satisfy the generalized curvature dimension inequality $C D\left(0, \rho_{2}, \kappa, d\right)$, for some values of the parameters $\rho_{2}$ and $\kappa$.

\section{The case $\rho_{1}=0$}

Throughout this section, we assume that $L$ satisfies the generalized curvature dimension inequality $C D\left(0, \rho_{2}, \kappa, d\right)$ with $\rho_{2}>0$ and $\kappa \geq 0$.

The main tool to prove the theorems mentioned in the introduction, is the heat semigroup $P_{t}=e^{t L}$, which is defined using the spectral theorem. Since $L$ satisfies the curvature dimension inequality, this semigroup is stochastically complete (see [3]), i.e. $P_{t} 1=1$. Moreover, thanks to the hypoellipticity of $L$, for $f \in L^{p}(\mathbb{M})$, $1 \leq p \leq \infty$, the function $(t, x) \rightarrow P_{t} f(x)$ is smooth on $\mathbb{M} \times(0, \infty)$ and

$$
P_{t} f(x)=\int_{\mathbb{M}} p(x, y, t) f(y) d \mu(y)
$$

where $p(x, y, t)=p(y, x, t)>0$ is the so-called heat kernel associated to $P_{t}$.

A key ingredient in the following analysis is the following gradient bound that was proved in [3].

Theorem 2.1 (Li-Yau type gradient estimate with $\rho_{1}=0$ ). Let $f \in C_{0}^{\infty}(\mathbb{M})$, $f \geq 0, f \neq 0$, then the following inequality holds for $t>0$ :

$$
\Gamma\left(\ln P_{t} f\right) \leq\left(1+\frac{3 \kappa}{2 \rho_{2}}\right) \frac{L P_{t} f}{P_{t} f}+\frac{d\left(1+3 \kappa /\left(2 \rho_{2}\right)\right)^{2}}{2 t} .
$$

\subsection{Gradient bounds for the heat semigroup}

Proposition 2.2. Let $f \in C_{0}^{\infty}(\mathbb{M})$.

- If $1 \leq p<2$, then for every $t>0$,

$$
\left\|\sqrt{\Gamma\left(P_{t} f\right)}\right\|_{p} \leq \frac{1+3 \kappa /\left(2 \rho_{2}\right)}{\sqrt{1+(p-1)\left(1+3 \kappa /\left(2 \rho_{2}\right)\right)}} \sqrt{\frac{d}{2 t}}\|f\|_{p} .
$$

- If $2 \leq p \leq+\infty$, then for every $t>0$,

$$
\left\|\sqrt{\Gamma\left(P_{t} f\right)}\right\|_{p} \leq \sqrt{\frac{1+2 \kappa / \rho_{2}}{2 t}}\|f\|_{p} .
$$

Proof. Suppose that $1 \leq p<2$. By Theorem 2.1, for $f \in C_{0}^{\infty}(\mathbb{M}), f \geq 0, f \not \equiv 0$, and $t>0$,

$$
\left(P_{t} f\right)^{p-2} \Gamma\left(P_{t} f\right) \leq \frac{D}{d}\left(P_{t} f\right)^{p-1}\left(L P_{t} f\right)+\frac{D^{2}}{2 t d}\left(P_{t} f\right)^{p},
$$


where $D=d\left(1+3 \kappa /\left(2 \rho_{2}\right)\right)$. It follows that

$$
\begin{aligned}
\int_{\mathbb{M}}\left(P_{t} f\right)^{p-2} \Gamma\left(P_{t} f\right) d \mu & \leq \frac{D}{d} \int_{\mathbb{M}}\left(P_{t} f\right)^{p-1}\left(L P_{t} f\right) d \mu+\frac{D^{2}}{2 t d} \int_{\mathbb{M}}\left(P_{t} f\right)^{p} d \mu \\
& =-\frac{D}{d} \int_{\mathbb{M}} \Gamma\left(\left(P_{t} f\right)^{p-1}, P_{t} f\right) d \mu+\frac{D^{2}}{2 t d} \int_{\mathbb{M}}\left(P_{t} f\right)^{p} d \mu \\
& =-\frac{D}{d} \int_{\mathbb{M}}(p-1)\left(P_{t} f\right)^{p-2} \Gamma\left(P_{t} f\right) d \mu+\frac{D^{2}}{2 t d} \int_{\mathbb{M}}\left(P_{t} f\right)^{p} d \mu .
\end{aligned}
$$

Observing $\int_{\mathbb{M}}\left(P_{t} f\right)^{p} d \mu=\left\|P_{t} f\right\|_{p}^{p} \leq\|f\|_{p}^{p}$, we get

$$
\int_{\mathbb{M}}\left(P_{t} f\right)^{p-2} \Gamma\left(P_{t} f\right) d \mu \leq \frac{1}{1+(p-1) D / d}\left(\frac{D^{2}}{2 t d}\right)\|f\|_{p}^{p} .
$$

On the other hand, let us choose $\alpha=p / 2$ and $\beta=(2-p) / 2$. Since $1 \leq p<2$, one can easily check that

$$
\begin{gathered}
\left(\int_{\mathbb{M}}\left(P_{t} f\right)^{p-2} \Gamma\left(P_{t} f\right) d \mu\right)^{\alpha}=\left\|\left(P_{t} f\right)^{p(p-2) / 2} \Gamma\left(P_{t} f\right)^{p / 2}\right\|_{2 / p}, \\
\left(\int_{\mathbb{M}}\left(P_{t} f\right)^{p} d \mu\right)^{\beta}=\left\|\left(P_{t} f\right)^{p(2-p) / 2}\right\|_{2 /(2-p)} .
\end{gathered}
$$

So, by Hölder's inequality, we obtain

$$
\int_{\mathbb{M}} \Gamma\left(P_{t} f\right)^{p / 2} d \mu \leq\left(\int_{\mathbb{M}}\left(P_{t} f\right)^{p-2} \Gamma\left(P_{t} f\right) d \mu\right)^{\alpha}\left(\int_{\mathbb{M}}\left(P_{t} f\right)^{p} d \mu\right)^{\beta},
$$

or, equivalently,

$$
\begin{aligned}
\int_{\mathbb{M}}\left(P_{t} f\right)^{p-2} & \Gamma\left(P_{t} f\right) d \mu \geq\left[\int_{\mathbb{M}} \Gamma\left(P_{t} f\right)^{p / 2} d \mu\left(\int_{\mathbb{M}}\left(P_{t} f\right)^{p} d \mu\right)^{-\beta}\right]^{1 / \alpha} \\
& =\left[\left\|\sqrt{\Gamma\left(P_{t} f\right)}\right\|_{p}^{p}\left\|P_{t} f\right\|_{p}^{-p \beta}\right]^{1 / \alpha}=\left[\left\|\sqrt{\Gamma\left(P_{t} f\right)}\right\|_{p}^{p}\left\|P_{t} f\right\|_{p}^{-p(2-p) / 2}\right]^{2 / p} \\
& =\left\|\sqrt{\Gamma\left(P_{t} f\right)}\right\|_{p}^{2}\left\|P_{t} f\right\|_{p}^{-(2-p)} .
\end{aligned}
$$

Therefore, for $1 \leq p<2$, we obtain

$$
\begin{aligned}
\left\|\sqrt{\Gamma\left(P_{t} f\right)}\right\|_{p}^{2} & \leq\left[\frac{1}{1+(p-1) D / d}\left(\frac{D^{2}}{2 t d}\right)\|f\|_{p}^{p}\right]\left\|P_{t} f\right\|_{p}^{2-p} \\
& \leq \frac{1}{1+(p-1) D / d}\left(\frac{D^{2}}{2 t d}\right)\|f\|_{p}^{p}\|f\|_{p}^{2-p}=\frac{1}{1+(p-1) D / d}\left(\frac{D^{2}}{2 t d}\right)\|f\|_{p}^{2} .
\end{aligned}
$$

For $f \in C_{0}^{\infty}(\mathbb{M})$, let us decompose $f$ as $f=f^{+}-f^{-}$, where $f^{+}=\max (f, 0)$ and $f^{-}=-\min (f, 0)$.

Then for each of $f^{+}$and $f^{-}$, the above gradient estimate holds.

We can then finish the proof by observing that $\|f\|_{p}=\left\|f^{+}\right\|_{p}+\left\|f^{-}\right\|_{p}$ and

$$
\left\|\sqrt{\Gamma\left(P_{t} f\right)}\right\|_{p} \leq\left\|\sqrt{\Gamma\left(P_{t} f^{+}\right)}+\sqrt{\Gamma\left(P_{t} f^{-}\right)}\right\|_{p} \leq\left\|\sqrt{\Gamma\left(P_{t} f^{+}\right)}\right\|_{p}+\left\|\sqrt{\Gamma\left(P_{t} f^{-}\right)}\right\|_{p} .
$$


Now suppose that $2 \leq p \leq+\infty$.

In [1], the following reverse Poincaré inequality (Caccioppoli type inequality) is proved:

$$
\Gamma\left(P_{t} f\right)+\rho_{2} t \Gamma^{Z}\left(P_{t} f\right) \leq \frac{1+2 \kappa / \rho_{2}}{2 t}\left(P_{t}\left(f^{2}\right)-\left(P_{t} f\right)^{2}\right)
$$

For $2 \leq p \leq+\infty$, one can write $\left\|P_{t}\left(f^{2}\right)\right\|_{p / 2} \leq\left\|f^{2}\right\|_{p / 2}=\|f\|_{p}^{2}$.

Therefore, we have

$$
\left\|\Gamma\left(P_{t} f\right)\right\|_{p / 2} \leq \frac{1+2 \kappa / \rho_{2}}{2 t}\left\|P_{t}\left(f^{2}\right)\right\|_{p / 2} \leq \frac{1+2 \kappa / \rho_{2}}{2 t}\|f\|_{p}^{2},
$$

which implies

$$
\left\|\sqrt{\Gamma\left(P_{t} f\right)}\right\|_{p} \leq \sqrt{\frac{1+2 \kappa / \rho_{2}}{2 t}}\|f\|_{p}
$$

\subsection{Pseudo-Poincaré inequalities}

By duality, the previous gradient bounds lead to the following pseudo-Poincaré type inequalities.

Proposition 2.3. Let $f \in C_{0}^{\infty}(\mathbb{M})$.

- If $1 \leq p<2$, then for every $t \geq 0$,

$$
\left\|f-P_{t} f\right\|_{p} \leq \sqrt{\left(2+\frac{4 \kappa}{\rho_{2}}\right) t}\|\sqrt{\Gamma(f)}\|_{p} .
$$

- If $2 \leq p \leq+\infty$, then for every $t \geq 0$,

$$
\left\|f-P_{t} f\right\|_{p} \leq \frac{\left(1+3 \kappa /\left(2 \rho_{2}\right)\right) \sqrt{2 d}}{\sqrt{1+(p-1)\left(1+3 \kappa /\left(2 \rho_{2}\right)\right)}} \sqrt{t}\|\sqrt{\Gamma(f)}\|_{p} .
$$

Proof. Let $p^{\prime}=p /(p-1)$. For any $g \in C_{0}^{\infty}(\mathbb{M})$ with $\|g\|_{p^{\prime}} \leq 1$, we have

$$
\begin{aligned}
\int_{\mathbb{M}} g\left(f-P_{t} f\right) d \mu & =\int_{\mathbb{M}} g\left(-\int_{0}^{t} \partial_{s} P_{s} f d s\right) d \mu=-\int_{0}^{t} \int_{\mathbb{M}} g L P_{s} f d \mu d s \\
& =-\int_{0}^{t} \int_{\mathbb{M}} g P_{s} L f d \mu d s=-\int_{0}^{t} \int_{\mathbb{M}} P_{s} g L f d \mu d s \\
& =\int_{0}^{t} \int_{\mathbb{M}} \Gamma\left(P_{s} g, f\right) d \mu d s \leq\|\sqrt{\Gamma(f)}\|_{p} \int_{0}^{t}\left\|\sqrt{\Gamma\left(P_{s} g\right)}\right\|_{p^{\prime}} d s .
\end{aligned}
$$

By using Proposition 2.2, we have

$$
\int_{0}^{t}\left\|\sqrt{\Gamma\left(P_{s} g\right)}\right\|_{p^{\prime}} d s \leq \int_{0}^{t} \frac{C_{p^{\prime}}}{\sqrt{s}} d s\|g\|_{p^{\prime}} .
$$


We therefore obtain

$$
\int_{\mathbb{M}} g\left(f-P_{t} f\right) d \mu \leq 2 C_{p^{\prime}} \sqrt{t}\|\sqrt{\Gamma(f)}\|_{p}\|g\|_{p^{\prime}}
$$

By duality we can now conclude that

$$
\left\|f-P_{t} f\right\|_{p} \leq 2 C_{p^{\prime}} \sqrt{t}\|\sqrt{\Gamma(f)}\|_{p}
$$

\subsection{Improved Sobolev embedding}

For $\alpha<0$, we define the Besov norm $\|\cdot\|_{B_{\infty, \infty}^{\alpha}}$ on $\mathbb{M}$ by

$$
\|f\|_{B_{\infty, \infty}^{\alpha}}=\sup _{t>0} t^{-\alpha / 2}\left\|P_{t} f\right\|_{\infty}
$$

It is clear from this definition that $\|f\|_{B_{\infty, \infty}^{\alpha}} \leq 1$ is equivalent to the fact that for every $u>0,\left|P_{t_{u}} f\right| \leq u$, where $t_{u}=u^{2 / \alpha}$. For $p \geq 1$, we then define the Sobolev space $W^{1, p}(\mathbb{M})$ as the closure of $C_{0}^{\infty}(\mathbb{M})$ with respect to the norm $\|f\|_{p}+\|\sqrt{\Gamma(f)}\|_{p}$.

Theorem 2.4 (Improved Sobolev embedding). For every $1 \leq p<q<\infty$ and every $f \in W^{1, p}(\mathbb{M})$, we have

$$
\|f\|_{q} \leq C\|\sqrt{\Gamma(f)}\|_{p}^{\theta}\|f\|_{B_{\infty, \infty}^{\theta /(\theta-1)}}^{1-\theta}
$$

where $\theta=p / q$ and where $C>0$ is a constant that only depends on $p, q, \rho_{2}, \kappa$ and $d$.

Proof. The techniques of the proof are mainly based on [14]; for the sake of completeness, we reproduce the main arguments and make sure they adapt to our sub-Riemannian framework. The proof proceeds in three steps.

Step 1. We first prove the weak-type inequality

$$
\|f\|_{q, \infty} \leq C\|\sqrt{\Gamma(f)}\|_{p}^{\theta}\|f\|_{\substack{B_{\infty, \infty}^{\theta /(\theta-1)} \\ 1-\theta}}^{\theta}
$$

Without loss of generality, we can assume $\|f\|_{B_{\infty, \infty}^{\theta /(\theta-1)}} \leq 1$, which is equivalent to the condition

$$
\left|P_{t_{u}} f\right| \leq u, t_{u}=u^{2(\theta-1) / \theta} \quad \text { for every } u>0 .
$$

We have then

$$
u^{q} \mu\{|f|>2 u\} \leq u^{q} \mu\left\{\left|f-P_{t_{u}} f\right|>u\right\} \leq u^{q-p} \int_{M}\left|f-P_{t_{u}} f\right|^{p} d \mu .
$$

From Proposition 2.3, we have

$$
\left\|f-P_{t} f\right\|_{p} \leq C \sqrt{t}\|\sqrt{\Gamma(f)}\|_{p} .
$$


Since $q-p+\frac{p}{2} \frac{2(\theta-1)}{\theta}=0$, we conclude

$$
u^{q} \mu\{|f|>2 u\} \leq u^{q-p}\left(C^{p} t_{u}^{p / 2}\|\sqrt{\Gamma(f)}\|_{p}^{p}\right) \leq C^{p}\|\sqrt{\Gamma(f)}\|_{p}^{p} .
$$

We finally observe that $\sup _{u>0} u^{q} \mu\{|f|>2 u\}=\frac{1}{2^{q}}\|f\|_{q, \infty}^{q}$, to conclude Step 1 .

Step 2. In the previous weak type inequality, we would like to replace the $L^{q, \infty}$ norm by the $L^{q}$-norm. Again, we assume $\|f\|_{B_{\infty, \infty}^{\theta /(\theta-1)}} \leq 1$. That is, $\left|P_{t_{u}} f\right| \leq u$ for $t_{u}=u^{2(\theta-1) / \theta}$, for all $u>0$. For $f \in W^{1, p}(\mathbb{M}) \cap L^{q}(\mathbb{M})$ such that $\left|P_{t_{u}} f\right| \leq u$, for all $u>0$, we want to show that for some constant $C>0$,

$$
\int_{M}|f|^{q} d \mu \leq C \int_{\mathbb{M}} \Gamma(f)^{p / 2} d \mu .
$$

Let $c \geq 5$ be an arbitrary constant. For any $u>0$, we introduce the truncation

$$
\tilde{f}_{u}=(f-u)^{+} \wedge((c-1) u)+(f+u)^{-} \vee(-(c-1) u) .
$$

That is, $\tilde{f}_{u}(x)=f(x)-u$ when $u \leq f(x) \leq c u$, and $\tilde{f}_{u}(x)=f(x)+u$ when $-c u \leq f(x) \leq-u$; otherwise $\left|\tilde{f}_{u}\right|$ is truncated as constants 0 or $(c-1) u$. Observing

$$
\{|f| \geq 5 u\} \subset\left\{\left|\tilde{f}_{u}\right| \geq 4 u\right\}
$$

yields

$$
\begin{aligned}
\int_{0}^{\infty} & \mu(\{|f| \geq 5 u\}) d\left(u^{q}\right) \leq \int_{0}^{\infty} \mu\left(\left\{\left|\tilde{f}_{u}\right| \geq 4 u\right\}\right) d\left(u^{q}\right) \\
& \leq \int_{0}^{\infty} \mu\left(\left\{\left|\tilde{f}_{u}-P_{t_{u}} f\right| \geq 3 u\right\}\right) d\left(u^{q}\right) \quad\left(\text { since }\left|P_{t_{u}}(f)\right| \leq u\right) \\
& \leq \int_{0}^{\infty} \mu\left(\left\{\left|\tilde{f}_{u}-P_{t_{u}} \tilde{f}_{u}\right| \geq u\right\}\right) d\left(u^{q}\right)+\int_{0}^{\infty} \mu\left(\left\{P_{t_{u}}\left(\left|f-\tilde{f}_{u}\right|\right) \geq 2 u\right\}\right) d\left(u^{q}\right) .
\end{aligned}
$$

We now apply the pseudo-Poincaré inequality for $\tilde{f}_{u}$ as follows:

$$
\begin{aligned}
\mu\left(\left\{\left|\tilde{f}_{u}-P_{t_{u}} \tilde{f}_{u}\right|\right.\right. & \geq u\}) \leq u^{-p} \int_{\mathbb{M}}\left|\tilde{f}_{u}-P_{t_{u}} \tilde{f}_{u}\right|^{p} d \mu \\
& \leq C^{\prime} u^{-p} t_{u}^{p / 2} \int_{\mathbb{M}} \Gamma\left(\tilde{f}_{u}\right)^{p / 2} d \mu=C^{\prime} u^{-q} \int_{\{u \leq|f| \leq c u\}} \Gamma(f)^{p / 2} d \mu .
\end{aligned}
$$

So, by integration, we get

$$
\begin{gathered}
\int_{0}^{\infty} \mu\left(\left\{\left|\tilde{f}_{u}-P_{t_{u}} \tilde{f}_{u}\right| \geq u\right\}\right) d\left(u^{q}\right) \leq \int_{0}^{\infty} C^{\prime} q u^{-1} \int_{\{u \leq|f| \leq c u\}} \Gamma(f)^{p / 2} d \mu d u \\
\leq C^{\prime} q \int_{\mathbb{M}} \Gamma(f)^{p / 2} \int_{|f| / c}^{|f|} \frac{d u}{u} d \mu=C^{\prime} q \ln c \int_{\mathbb{M}} \Gamma(f)^{p / 2} d \mu .
\end{gathered}
$$


On the other hand, we have

$$
\begin{aligned}
\left|f-\tilde{f}_{u}\right| & =\left|f-\tilde{f}_{u}\right| 1_{\{|f| \leq c u\}}+\left|f-\tilde{f}_{u}\right| 1_{\{|f|>c u\}} \\
& =\min (u,|f|) 1_{\{|f| \leq c u\}}+(|f|-(c-1) u) 1_{\{|f|>c u\}} \leq u+|f| 1_{\{|f|>c u\}} .
\end{aligned}
$$

By integrating, we then obtain

$$
\begin{aligned}
\int_{0}^{\infty} \mu( & \left.\left\{P_{t_{u}}\left(\left|f-\tilde{f}_{u}\right|\right) \geq 2 u\right\}\right) d\left(u^{q}\right) \leq \int_{0}^{\infty} \mu\left(\left\{P_{t_{u}}\left(|f| 1_{\{|f|>c u\}}\right) \geq u\right\}\right) d\left(u^{q}\right) \\
& \leq \int_{0}^{\infty} \frac{1}{u}\left(\int_{\mathbb{M}}\left(|f| 1_{\{|f|>c u\}}\right) d \mu\right) d\left(u^{q}\right) \quad\left(P_{t} \text { is a contraction on } L^{1}(\mathbb{M})\right) \\
& =\frac{q}{q-1} \int_{\mathbb{M}}|f|\left(\int_{0}^{\infty} 1_{\{|f|>c u\}} d\left(u^{q-1}\right)\right) d \mu=\frac{q}{q-1} \frac{1}{c^{q-1}}\|f\|_{q}^{q} .
\end{aligned}
$$

Gathering all the estimates, we can then conclude

$$
\begin{aligned}
\frac{1}{5^{q}} \int_{\mathbb{M}}|f|^{q} d \mu & =\frac{1}{5^{q}}\|f\|_{q}^{q}=\int_{0}^{\infty} \mu(\{|f| \geq 5 u\}) d\left(u^{q}\right) \\
& \leq C^{\prime} q \ln c \int_{\mathbb{M}} \Gamma(f)^{p / 2} d \mu+\frac{q}{q-1} \frac{1}{c^{q-1}}\|f\|_{q}^{q}
\end{aligned}
$$

If we pick a large $c \geq 5$ depending on $q$ such that $\frac{1}{5^{q}}>\frac{q}{q-1} \frac{1}{c^{q-1}}$, we have proved

$$
\|f\|_{q}^{q} \leq C^{q}\|\sqrt{\Gamma(f)}\|^{p}
$$

with

$$
C=\left(\frac{C^{\prime} q \ln c}{\frac{1}{5^{q}}-\frac{q}{(q-1) c^{q-1}}}\right)^{1 / q}
$$

Step 3. Finally, it remains to prove that $\|f\|_{q}<\infty$ is actually a consequence of $\|\sqrt{\Gamma(f)}\|_{p}<\infty$ and $\|f\|_{B_{\infty, \infty}^{\theta /(\theta-1)}} \leq 1$, so that we can remove the condition $f \in L^{q}(\mathbb{M})$ from Step 2 and complete the proof of theorem. From the weak type inequality of Step 1 , we have $\|f\|_{q, \infty}<\infty$. For any $0<\epsilon<1$, we define

$$
N_{\epsilon}(f)=\int_{\epsilon}^{1 / \epsilon} \mu(\{|f| \geq 5 u\}) d\left(u^{q}\right) \leq \frac{2 q}{5^{q}}\left(\ln \frac{1}{\epsilon}\right)\|f\|_{q, \infty}^{q}<\infty .
$$

Following the argument in Step 2 again, we see that

$$
N_{\epsilon}(f) \leq C^{\prime} q \ln c \int_{\mathbb{M}} \Gamma(f)^{p / 2} d \mu+\int_{\epsilon}^{1 / \epsilon} \frac{1}{u}\left(\int_{\mathbb{M}}\left(|f| 1_{\{|f|>c u\}}\right) d \mu\right) d\left(u^{q}\right) .
$$


The first term is bounded, and the second term can be estimated as follows:

$$
\begin{aligned}
& \int_{\epsilon}^{1 / \epsilon} \frac{1}{u}\left(\int_{\mathbb{M}}\left(|f| 1_{\{|f|>c u\}}\right) d \mu\right) d\left(u^{q}\right) \\
& =\int_{\epsilon}^{1 / \epsilon} \frac{1}{u}\left(c u \mu(\{|f|>c u\})+c \int_{u}^{\infty} \mu(\{|f|>c v\}) d v\right) d\left(u^{q}\right) \\
& \leq\left(c+\frac{c}{q-1}\right) \int_{\epsilon}^{1 / \epsilon} \mu(\{|f| \geq c u\}) d\left(u^{q}\right)+\frac{c q}{(q-1) \epsilon^{q-1}} \int_{1 / \epsilon}^{\infty} \mu(\{|f| \geq c u\}) d u \\
& \leq \frac{q}{q-1} \frac{5^{q}}{c^{q-1}} N_{\epsilon}(f)+\frac{c q}{q-1} \int_{5 / c \epsilon}^{1 / \epsilon} \frac{\|f\|_{q, \infty}^{q}}{(c u)^{q}} d\left(u^{q}\right)+\frac{c q}{(q-1) \epsilon^{q-1}} \int_{1 / \epsilon}^{\infty} \frac{\|f\|_{q, \infty}^{q}}{(c u)^{q}} d u \\
& =\frac{q}{q-1} \frac{5^{q}}{c^{q-1}} N_{\epsilon}(f)+\frac{q}{q-1} \frac{1}{c^{q-1}}\|f\|_{q, \infty}^{q}\left(q \ln \frac{c}{5}+\frac{1}{q-1}\right) .
\end{aligned}
$$

So, by choosing $c$ large enough, we have $\sup _{0<\epsilon<1} N_{\epsilon}(f)<\infty$, which implies $\|f\|_{q}=\lim _{\epsilon \rightarrow 0} 5\left(N_{\epsilon}(f)\right)^{1 / q}<\infty$. This completes the proof.

\subsection{Sobolev inequality, isoperimetry and volume growth}

In this section, we study the Sobolev and isoperimetric inequalities and their connections with the volume growth of metric balls. We obtain the sub-Riemannian analogue of a theorem essentially due to Ledoux [13].

We first recall what we mean by the perimeter of a set in our subelliptic setting. For further details, we refer to [8].

Let us first observe that, given any point $x \in \mathbb{M}$ there exists an open set $x \in U \subset \mathbb{M}$ in which the operator $L$ can be written as

$$
L=-\sum_{i=1}^{m} X_{i}^{*} X_{i}
$$

where the vector fields $X_{i}$ have Lipschitz continuous coefficients in $U$, and $X_{i}^{*}$ indicates the formal adjoint of $X_{i}$ in $L^{2}(\mathbb{M}, d \mu)$.

We indicate by $\mathcal{F}(\mathbb{M})$ the set of $C^{1}$ vector fields which are subunit for $L$. Given a function $f \in L_{\text {loc }}^{1}(\mathbb{M})$, which is supported in $U$ we define the horizontal total variation of $f$ as

$$
\operatorname{Var}(f)=\sup _{\phi \in \mathcal{F}(\mathbb{M})} \int_{U} f\left(\sum_{i=1}^{m} X_{i}^{*} \phi_{i}\right) d \mu,
$$

where on $U, \phi=\sum_{i=1}^{m} \phi_{i} X_{i}$. For functions not supported in $U$, $\operatorname{Var}(f)$ may be defined by using a partition of unity. The space

$$
B V(\mathbb{M})=\left\{f \in L^{1}(\mathbb{M}) \mid \operatorname{Var}(f)<\infty\right\}
$$

endowed with the norm

$$
\|f\|_{B V(\mathbb{M})}=\|f\|_{L^{1}(\mathbb{M})}+\operatorname{Var}(f)
$$


is a Banach space. It is well known that $W^{1,1}(\mathbb{M})=\left\{f \in L^{1}(\mathbb{M}) \mid \sqrt{\Gamma f} \in L^{1}(\mathbb{M})\right\}$ is a strict subspace of $B V(\mathbb{M})$ and when $f \in W^{1,1}(\mathbb{M})$ one has in fact

$$
\operatorname{Var}(f)=\|\sqrt{\Gamma(f)}\|_{L^{1}(\mathbb{M})} .
$$

Given a measurable set $E \subset \mathbb{M}$ we say that it has finite perimeter, or is a Cacciopoli set if $\mathbf{1}_{E} \in B V(\mathbb{M})$. In this case the perimeter of $E$ is, by definition,

$$
P(E)=\operatorname{Var}\left(\mathbf{1}_{E}\right)
$$

In a later section, we will need the following approximation result, see Theorem 1.14 in [8].

Lemma 2.5. Let $f \in B V(\mathbb{M})$. Then there exists a sequence $\left\{f_{n}\right\}_{n \in \mathbb{N}}$ of functions in $C_{0}^{\infty}(\mathbb{M})$ such that:

(i) $\left\|f_{n}-f\right\|_{L^{1}(\mathbb{M})} \rightarrow 0$;

(ii) $\int_{\mathbb{M}} \sqrt{\Gamma\left(f_{n}\right)} d \mu \rightarrow \operatorname{Var}(f)$.

We now prove the main result of this subsection.

Theorem 2.6. Let $D>1$. Assume that $\mathbb{M}$ is not compact in the metric topology. Then the following assertions are equivalent:

(1) There exists a constant $C_{1}>0$ such that for every $x \in \mathbb{M}$ and $r \geq 0$,

$$
\mu(B(x, r)) \geq C_{1} r^{D} .
$$

(2) There exists a constant $C_{2}>0$ such that for $x \in \mathbb{M}$ and $t>0$,

$$
p(x, x, t) \leq \frac{C_{2}}{t^{D / 2}}
$$

(3) For some $1 \leq p, q, r<\infty$ with $1 / q=1 / p-r /(q D)$, there exists a constant $C_{3}>0$ such that for all $f \in C_{0}^{\infty}(\mathbb{M})$, we have

$$
\|f\|_{q} \leq C_{3}\|\sqrt{\Gamma(f)}\|_{p}^{p / q}\|f\|_{r}^{1-p / q} .
$$

(4) There exists a constant $C_{4}>0$ such that, for every Caccioppoli set $E \subset \mathbb{M}$, one has

$$
\mu(E)^{(D-1) / D} \leq C_{4} P(E)
$$

Remark 2.7. If we replace the condition of (3) by for all $1 \leq p, q, r<\infty$ with $1 / q=1 / p-r /(q D),(1),(2),(3)$ and (4) would still be equivalent. 
Proof. That $(1) \rightarrow(2)$ follows immediately from the Li-Yau Gaussian upper bound

$$
p(x, x, t) \leq \frac{C}{\mu(B(x, \sqrt{t})}
$$

that is proved in [3].

The proof that $(2) \rightarrow(3)$ follows from the improved Sobolev embedding Theorem 2.4.

Indeed, (2) implies first that for $x, y \in \mathbb{M}$,

$$
\begin{aligned}
p(x, y, t) & =\int_{\mathbb{M}} p(x, z, t / 2) p(z, y, t / 2) \mu(d y) \\
& \leq\left(\int_{\mathbb{M}} p(x, z, t / 2)^{2} \mu(d z)\right)^{1 / 2}\left(\int_{\mathbb{M}} p(y, z, t / 2)^{2} \mu(d z)\right)^{1 / 2} \\
& =\sqrt{p(x, x, t) p(y, y, t)} \leq \frac{C_{2}}{t^{D / 2}} .
\end{aligned}
$$

Therefore, for every $f \in L^{1}(\mathbb{M})$, we have

$$
\left\|P_{t}(f)\right\|_{\infty}=\left\|\int_{\mathbb{M}} p(\cdot, y, t) f(y) \mu(d y)\right\|_{\infty} \leq\|p(\cdot, y, t)\|_{\infty}\|f\|_{1} \leq \frac{C_{2}}{t^{D / 2}}\|f\|_{1} .
$$

On the other hand, $P_{t}$ is a contraction on $L^{\infty}(\mathbb{M})$, i.e. $\left\|P_{t}\right\|_{\infty \rightarrow \infty} \leq 1$. Therefore, by the Riesz-Thorin interpolation theorem, we deduce that we have the following heat semigroup embedding:

$$
\left\|P_{t}\right\|_{r \rightarrow \infty} \leq \frac{C_{2}^{1 / r}}{t^{D /(2 r)}}, \quad r \geq 1 .
$$

Let now $1 \leq p, q, r<\infty$ be such that $1 / q=1 / p-r /(q D)$. Since for $\theta=p / q$, $-\theta /(2(\theta-1))-D /(2 r)=0$, we have

$$
\begin{aligned}
\|f\|_{B_{\infty}^{\theta, \infty}}^{\theta /(\theta-1)} & =\sup _{t>0} t^{-\theta /(2(\theta-1))}\left\|P_{t} f\right\|_{\infty} \\
& \leq \sup _{t>0} t^{-\theta /(2(\theta-1))} \frac{C_{2}^{1 / r}}{t^{D /(2 r)}}\|f\|_{r}=C_{2}^{1 / r}\|f\|_{r},
\end{aligned}
$$

we can conclude (3) from the improved Sobolev embedding of Theorem 2.4.

The proof that (3) is equivalent to (4) follows the classical ideas of FlemingRishel and Maz'ya, and it is based on a generalization of Federer's co-area formula for the space $B V(\mathbb{M})$, see for instance [8].

Finally, we show that $(3) \rightarrow(1)$. We adapt an idea in [16] (see Theorem 3.1.5 on p. 58). For any fixed $x \in \mathbb{M}$ and $s>0$, consider the function

$$
f(y)=\max \{s-d(x, y), 0\} .
$$

Then, it is easily seen that

$$
\begin{aligned}
\|f\|_{q} & \geq(s / 2) \mu(B(x, s / 2))^{1 / q} \\
\|f\|_{r} & \leq s \mu(B(x, s))^{1 / r} \\
\|\sqrt{\Gamma(f)}\|_{p} & \leq \mu(B(x, s))^{1 / p} .
\end{aligned}
$$


Hence, from (3) we have

$$
\begin{aligned}
\mu(B(x, s / 2))^{1 / q} & \leq 2 C_{3} s^{-p / q} \mu(B(x, s))^{1 / q+(1 / r)(1-p / q)} \\
& =2 C_{3} s^{-p / q} \mu(B(x, s))^{1 / q+p /(q D)} .
\end{aligned}
$$

This can be written as

$$
\begin{aligned}
& \mu(B(x, s)) \geq\left(2 C_{3}\right)^{-D q /(D+p)} s^{D p /(D+p)} \mu(B(x, s / 2))^{D /(D+p)} . \\
& \mu(B(x, s)) \geq\left\{\left(2 C_{3}\right)^{-q} s^{p}\right\}^{a} \mu(B(x, s / 2))^{a}
\end{aligned}
$$

where $a=D /(D+p)<1$. Replacing $s$ by $s / 2$ iteratively, we obtain

$$
\mu(B(x, s)) \geq\left(2 C_{3}\right)^{-q\left(\sum_{j=1}^{i} a^{j}\right)} s^{p\left(\sum_{j=1}^{i} a^{j}\right)} 2^{-p\left(\sum_{j=1}^{i}(j-1) a^{j}\right)} \mu\left(B\left(x, s / 2^{i}\right)\right)^{a^{i}} .
$$

From the volume doubling property proved in [2], we have the control

$$
\mu\left(B\left(x, s / 2^{i}\right)\right) \geq C^{-1}\left(1 / 2^{i}\right)^{Q} \mu(B(x, s)),
$$

for some $C=C\left(\rho_{1}, \rho_{2}, \kappa, d\right)>0$ and $Q=\log _{2} C$.

Therefore, we have

$$
\liminf _{i \rightarrow \infty} \mu\left(B\left(x, s / 2^{i}\right)\right)^{a^{i}} \geq \lim _{i \rightarrow \infty}\left(C^{-1} \mu(B(x, s))\right)^{a^{i}}(1 / 2)^{i Q a^{i}}=1 .
$$

Since $\sum_{j=1}^{\infty} a^{j}=D / p, \sum_{j=1}^{\infty}(j-1) a^{j}=D^{2} / p^{2}$, we obtain the volume growth control

$$
\mu(B(x, s)) \geq 2^{-(q+D) D / p} C_{3}^{-q D / p} s^{D} .
$$

This establishes (1), thus completing the proof.

Remark 2.8. By combining the results of [2] and [8], an alternative proof of $(1) \rightarrow(4)$ could be given. Indeed, in [8] it was proved that in a Carnot-Carathéodory space $(X, \mu, d)$ the doubling condition

$$
\mu(B(x, 2 r)) \leq C_{1} \mu(B(x, r)), \quad x \in X, r>0,
$$

for the volume of the metric balls combined with a weak Poincaré inequality suffices to establish the basic relative isoperimetric inequality

$$
\begin{array}{r}
\min \{\mu(E \cap B(x, r)), \mu((X \backslash E) \cap B(x, r))\}^{(D-1) / D} \\
\leq C_{\text {iso }}\left(\frac{r^{D}}{\mu(B(x, r))}\right)^{1 / D} P(E, B(x, r)),
\end{array}
$$

where $E \subset X$ is any set of locally finite perimeter. In this inequality the number $D=\log _{2} C_{1}$, where $C_{1}$ is the doubling constant, and $C_{\text {iso }}$ is a constant which depends only on $C_{1}$ and on the constant in the Poincaré inequality. If in addition the space $X$ satisfies the volume growth condition

$$
\mu(B(x, r)) \geq C_{2} r^{D}, \quad x \in \mathbb{M}, r>0,
$$


then (2.7) gives the global isoperimetric inequality

$$
\mu(E)^{(D-1) / D} \leq C_{\text {iso }} P(E, \mathbb{M})
$$

for any measurable set of locally finite perimeter $E \subset \mathbb{M}$. Since in [2], it was proved that the doubling condition and the weak Poincaré inequality are satisfied when $\rho_{1} \geq 0$, we conclude that $(1) \rightarrow(4)$.

\section{The case $\rho_{1}>0$}

Throughout this section, we assume that $L$ satisfies the generalized curvature dimension inequality $C D\left(\rho_{1}, \rho_{2}, \kappa, d\right)$ with $\rho_{1}>0, \rho_{2}>0$ and $\kappa \geq 0$. The following gradient bound was also proved in [3].

Theorem 3.1 (Li-Yau type gradient estimate with $\rho_{1}>0$ ). Let $f \in C_{0}^{\infty}(\mathbb{M})$, $f \geq 0$ and $f \not \equiv 0$. Then the following inequality holds for $t>0$ :

$$
\Gamma\left(\ln P_{t} f\right) \leq \frac{2 \rho_{2}+3 \kappa}{2 \rho_{2}} e^{-\frac{2 \rho_{1} \rho_{2}}{3\left(\rho_{2}+\kappa\right)} t} \frac{L P_{t} f}{P_{t} f}+\frac{d \rho_{1}}{12 \rho_{2}} \frac{\left(2 \rho_{2}+3 \kappa\right)^{2}}{\rho_{2}+\kappa} \frac{e^{-\frac{4 \rho_{1} \rho_{2}}{3\left(\rho_{2}+\kappa\right)} t}}{1-e^{-\frac{2 \rho_{1} \rho_{2}}{3\left(\rho_{2}+\kappa\right)} t}} .
$$

\subsection{Gradient bounds for the heat semigroup}

We first establish the following reverse Poincaré inequality.

Proposition 3.2. For $f \in C_{0}^{\infty}(\mathbb{M})$ and $t \geq 0$,

$$
\Gamma\left(P_{t} f\right) \leq \frac{1}{2} \rho_{1} \frac{\rho_{2}+2 \kappa}{\rho_{2}+\kappa} \frac{e^{-2 \frac{\rho_{1} \rho_{2}}{\rho_{2}+\kappa} t}}{1-e^{-\frac{\rho_{1} \rho_{2}}{\rho_{2}+\kappa} t}}\left(P_{t} f^{2}-\left(P_{t} f\right)^{2}\right) .
$$

Proof. Let us fix $T>0$ once and for all in the following proof. Given a function $f \in C_{0}^{\infty}(\mathbb{M})$, for $0 \leq t \leq T$ we introduce the functionals

$$
\phi_{1}(x, t)=\Gamma\left(P_{T-t} f\right)(x), \quad \text { and } \quad \phi_{2}(x, t)=\Gamma^{Z}\left(P_{T-t} f\right)(x),
$$

which are defined on $\mathbb{M} \times[0, T]$. A straightforward computation shows that

$$
L \phi_{1}+\frac{\partial \phi_{1}}{\partial t}=2 \Gamma_{2}\left(P_{T-t} f\right) . \quad \text { and } \quad L \phi_{2}+\frac{\partial \phi_{2}}{\partial t}=2 \Gamma_{2}^{Z}\left(P_{T-t} f\right) .
$$

Consider now the function

$$
\phi(x, t)=a(t) \phi_{1}(x, t)+b(t) \phi_{2}(x, t)=a(t) \Gamma\left(P_{T-t} f\right)(x)+b(t) \Gamma^{Z}\left(P_{T-t} f\right)(x),
$$

where $a$ and $b$ are two nonnegative functions that will be chosen later. Applying the generalized curvature dimension inequality $C D\left(\rho_{1}, \rho_{2}, \kappa, \infty\right)$, we obtain

$$
\begin{aligned}
L \phi+\frac{\partial \phi}{\partial t} & =a^{\prime} \Gamma\left(P_{T-t} f\right)+b^{\prime} \Gamma^{Z}\left(P_{T-t} f\right)+2 a \Gamma_{2}\left(P_{T-t} f\right)+2 b \Gamma_{2}^{Z}\left(P_{T-t} f\right) \\
& \geq\left(a^{\prime}+2 \rho_{1} a-2 \kappa \frac{a^{2}}{b}\right) \Gamma\left(P_{T-t} f\right)+\left(b^{\prime}+2 \rho_{2} a\right) \Gamma^{Z}\left(P_{T-t} f\right) .
\end{aligned}
$$


Let us now choose

$$
b(t)=\left(\exp \left(-\frac{2 \rho_{1} \rho_{2} t}{\kappa+\rho_{2}}\right)-\exp \left(-\frac{2 \rho_{1} \rho_{2} T}{\kappa+\rho_{2}}\right)\right)^{2} \quad \text { and } \quad a(t)=-\frac{b^{\prime}(t)}{2 \rho_{2}},
$$

so that

$$
b^{\prime}+2 \rho_{2} a=0 \quad \text { and } \quad a^{\prime}+2 \rho_{1} a-2 \kappa \frac{a^{2}}{b} \geq \rho_{1} \frac{\rho_{2}+2 \kappa}{\rho_{2}+\kappa} e^{-2 \frac{\rho_{1} \rho_{2}}{\rho_{2}+\kappa} T} .
$$

With this choice, we get

$$
L \phi+\frac{\partial \phi}{\partial t} \geq-\rho_{1} \frac{\rho_{2}+2 \kappa}{\rho_{2}+\kappa} e^{-2 \frac{\rho_{1} \rho_{2}}{\rho_{2}+\kappa} T} \Gamma\left(P_{T-t} f\right) .
$$

and therefore from a comparison theorem for parabolic partial differential equations (see [3]) we have

$$
P_{T}(\phi(\cdot, T))(x) \geq \phi(x, 0)-\rho_{1} \frac{\rho_{2}+2 \kappa}{\rho_{2}+\kappa} e^{-2 \frac{\rho_{1} \rho_{2}}{\rho_{2}+\kappa} T} \int_{0}^{T} P_{t}\left(\Gamma\left(P_{T-t} f\right)\right) d t .
$$

It is easily seen that

$$
\int_{0}^{T} P_{t}\left(\Gamma\left(P_{T-t} f\right)\right) d t=\frac{1}{2}\left(P_{T} f^{2}-\left(P_{T} f\right)^{2}\right)
$$

and, since,

$$
\phi(x, 0)=a(0) \Gamma\left(P_{T} f\right)(x)+b(0) \Gamma^{Z}\left(P_{T} f\right)(x)
$$

and

$$
P_{T}(\phi(\cdot, T))(x)=a(T) P_{T}(\Gamma(f))(x)+b(T) P_{T}\left(\Gamma^{Z}(f)\right)(x)=0,
$$

the proof is completed.

Proposition 3.3. Let $f \in C_{0}^{\infty}(\mathbb{M})$.

- If $1 \leq p<2$, then for every $t>0$,

$$
\left\|\sqrt{\Gamma\left(P_{t} f\right)}\right\|_{p} \leq\left(\frac{\frac{d \rho_{1} \rho_{2}}{3\left(\rho_{2}+\kappa\right)} \frac{\left(1+\frac{3 \kappa}{2 \rho_{2}}\right)^{2} e^{-\frac{4 \rho_{1} \rho_{2}}{3\left(\rho_{2}+\kappa\right)} t}}{\left(1-e^{-\frac{2 \rho_{1} \rho_{2}}{3\left(\rho_{2}+\kappa\right)} t}\right)}}{1+(p-1)\left(1+\frac{3 \kappa}{2 \rho_{2}}\right) e^{-\frac{2 \rho_{1} \rho_{2}}{3\left(\rho_{2}+\kappa\right)} t}}\right)^{1 / 2}\|f\|_{p} .
$$

- If $2 \leq p \leq+\infty$, then for every $t>0$,

$$
\left\|\sqrt{\Gamma\left(P_{t} f\right)}\right\|_{p} \leq\left(\frac{1}{2} \rho_{1} \frac{\rho_{2}+2 \kappa}{\rho_{2}+\kappa} \frac{e^{-2 \frac{\rho_{1} \rho_{2}}{\rho_{2}+\kappa} t}}{1-e^{-\frac{\rho_{1} \rho_{2}}{\rho_{2}+\kappa} t}}\right)^{1 / 2}\|f\|_{p} .
$$


Proof. The proof is essentially the same as the proof of Proposition 2.2. We observe from this proof that if for $f \in C_{0}^{\infty}(\mathbb{M})$ and $t>0$,

$$
\begin{aligned}
& \Gamma\left(\ln P_{t} f\right) \leq \alpha(t) \frac{L P_{t} f}{P_{t} f}+\beta(t), \quad f \geq 0, f \not \equiv 0, \alpha(t), \beta(t)>0, \\
& \Gamma\left(P_{t} f\right) \leq \gamma(t)\left(P_{t} f^{2}-\left(P_{t} f\right)^{2}\right), \quad \gamma(t)>0,
\end{aligned}
$$

then

$$
\begin{array}{cc}
\left\|\sqrt{\Gamma\left(P_{t} f\right)}\right\|_{p} \leq\left(\frac{\beta(t)}{1+(p-1) \alpha(t)}\right)^{1 / 2}\|f\|_{p}, & \text { for } 1 \leq p<2, \\
\left\|\sqrt{\Gamma\left(P_{t} f\right)}\right\|_{p} \leq(\gamma(t))^{1 / 2}\|f\|_{p}, & \text { for } 2 \leq p<\infty .
\end{array}
$$

By Theorem 3.1 and Proposition 3.2, we then see that $\alpha(t), \beta(t), \gamma(t)$ are given by

$$
\begin{aligned}
& \alpha(t)=\left(1+\frac{3 \kappa}{2 \rho_{2}}\right) e^{-\frac{2 \rho_{1} \rho_{2}}{3\left(\rho_{2}+\kappa\right)} t}, \quad \beta(t)=\frac{d \rho_{1} \rho_{2}}{3\left(\rho_{2}+\kappa\right)} \frac{\left(1+\frac{3 \kappa}{2 \rho_{2}}\right)^{2} e^{-\frac{4 \rho_{1} \rho_{2}}{3\left(\rho_{2}+\kappa\right)} t}}{\left(1-e^{-\frac{2 \rho_{1} \rho_{2}}{3\left(\rho_{2}+\kappa\right)} t}\right)} \\
& \gamma(t)=\frac{1}{2} \rho_{1} \frac{\rho_{2}+2 \kappa}{\rho_{2}+\kappa} \frac{e^{-2 \frac{\rho_{1} \rho_{2}}{\rho_{2}+\kappa} t}}{1-e^{-\frac{\rho_{1} \rho_{2}}{\rho_{2}+\kappa} t}} .
\end{aligned}
$$

\subsection{Pseudo-Poincaré inequalities}

Proposition 3.4. Let $f \in C_{0}^{\infty}(\mathbb{M})$.

- If $1 \leq p<2$, then for every $t \geq 0$,

$$
\left\|f-P_{t} f\right\|_{p} \leq\left(\frac{2\left(\rho_{2}+2 \kappa\right)\left(\rho_{2}+\kappa\right)}{\rho_{1} \rho_{2}^{2}}\left(1-e^{-\frac{\rho_{1} \rho_{2}}{\rho_{2}+\kappa} t}\right)\right)^{1 / 2}\|\sqrt{\Gamma(f)}\|_{p} .
$$

- If $2 \leq p \leq+\infty$, then for every $t \geq 0$,

$$
\left\|f-P_{t} f\right\|_{p} \leq\left(1+\frac{3 \kappa}{2 \rho_{2}}\right)\left(\frac{3 d\left(\rho_{2}+\kappa\right)}{\rho_{1} \rho_{2}}\left(1-e^{-\frac{2 \rho_{1} \rho_{2}}{3\left(\rho_{2}+\kappa\right)} t}\right)\right)^{1 / 2}\|\sqrt{\Gamma(f)}\|_{p}
$$

Proof. As shown in the proof of Proposition 2.3, we have

$$
\begin{array}{r}
\left\|f-P_{t} f\right\|_{p} \leq\left(\int_{0}^{t} \sqrt{\gamma(s)} d s\right)\|\sqrt{\Gamma(f)}\|_{p}, \quad \text { for } 1 \leq p<2, \\
\left\|f-P_{t} f\right\|_{p} \leq\left(\int_{0}^{t} \sqrt{\frac{\beta(s)}{1+(p-1) \alpha(s)}} d s\right)\|\sqrt{\Gamma(f)}\|_{p}, \quad \text { for } 2 \leq p<\infty,
\end{array}
$$


where $\alpha, \beta$ and $\gamma$ are defined in the proof of Proposition 3.3. The proof is finished by

$$
\begin{aligned}
\int_{0}^{t} \sqrt{\gamma(s)} d s & =\int_{0}^{t}\left(\frac{\rho_{1}\left(\rho_{2}+2 \kappa\right)}{2\left(\rho_{2}+\kappa\right)}\right)^{1 / 2} \frac{e^{-\frac{\rho_{1} \rho_{2}}{\rho_{2}+\kappa} s}}{\sqrt{1-e^{-\frac{\rho_{1} \rho_{2}}{\rho_{2}+\kappa}}}} d s \\
& =\left(\frac{2\left(\rho_{2}+2 \kappa\right)\left(\rho_{2}+\kappa\right)}{\rho_{1} \rho_{2}^{2}}\left(1-e^{-\frac{\rho_{1} \rho_{2}}{\rho_{2}+\kappa} t}\right)\right)^{1 / 2} \\
\int_{0}^{t} \sqrt{\frac{\beta(s)}{1+(p-1) \alpha(s)} d s} & \leq \int_{0}^{t} \sqrt{\beta(s)} d s \\
& =\int_{0}^{t}\left(\frac{d \rho_{1} \rho_{2}}{3\left(\rho_{2}+\kappa\right)} \frac{\left(1+\frac{3 \kappa}{2 \rho_{2}}\right)^{2} e^{-\frac{4 \rho_{1} \rho_{2}}{3\left(\rho_{2}+\kappa\right)}} s}{\left(1-e^{-\frac{2 \rho_{1} \rho_{2}}{3\left(\rho_{2}+\kappa\right)} s}\right)}\right)^{1 / 2} d s \\
& =\left(1+\frac{3 \kappa}{2 \rho_{2}}\right)\left(\frac{3 d\left(\rho_{2}+\kappa\right)}{\rho_{1} \rho_{2}}\left(1-e^{-\frac{2 \rho_{1} \rho_{2}}{3\left(\rho_{2}+\kappa\right)}} t\right)\right)^{1 / 2} .
\end{aligned}
$$

\subsection{Poincaré inequality}

In the case of $\rho_{1}>0$, we have the following theorem which is proved in [1].

Theorem 3.5. The measure $\mu$ is finite, i.e., $\mu(\mathbb{M})<+\infty$ and for every $1 \leq p \leq \infty$ and $f \in L^{p}(\mathbb{M})$,

$$
P_{t} f \stackrel{t \rightarrow \infty}{\longrightarrow} \frac{1}{\mu(\mathbb{M})} \int_{\mathbb{M}} f d \mu \text {. }
$$

This theorem allows us to deduce the Poincaré inequality.

Proposition 3.6. Let $1 \leq p<\infty$. There exists $C=C_{p}\left(\rho_{1}, \rho_{2}, \kappa, d\right)>0$ such that, for all $f \in C_{0}^{\infty}(\mathbb{M})$,

$$
\left\|f-f_{\mathbb{M}}\right\|_{p} \leq C\|\sqrt{\Gamma(f)}\|_{p}
$$

where $f_{\mathbb{M}}=\frac{1}{\mu(\mathbb{M})} \int_{\mathbb{M}} f d \mu$.

Proof. The proof is immediate from Proposition 3.4 and Theorem 3.5 by letting $t \rightarrow \infty$. And $C$ is given by

$$
C_{p}\left(\rho_{1}, \rho_{2}, \kappa, d\right)= \begin{cases}\left(\frac{2\left(\rho_{2}+2 \kappa\right)\left(\rho_{2}+\kappa\right)}{\rho_{1} \rho_{2}^{2}}\right)^{1 / 2} & \text { if } 1 \leq p<2, \\ \left(1+\frac{3 \kappa}{2 \rho_{2}}\right)\left(\frac{3 d\left(\rho_{2}+\kappa\right)}{\rho_{1} \rho_{2}}\right)^{1 / 2} & \text { if } 2 \leq p<\infty\end{cases}
$$

\subsection{A lower bound on the Cheeger's isoperimetric constant}

In [6], in order to bound from below the first eigenvalue $\lambda_{1}$ of a compact Riemannian manifold with normalized Riemannian measure $\mu_{g}$, Cheeger introduced the following isoperimetric constant:

$$
h=\inf \frac{\mu_{g}(\partial A)}{\mu_{g}(A)},
$$


where the infimum runs over all open subsets $A$ with smooth boundary $\partial A$ such that $\mu(A) \leq 1 / 2$. Cheeger's inequality then states $\lambda_{1} \geq h^{2} / 4$.

Such an isoperimetric quantity may also be considered and estimated in our sub-Riemannian framework. Throughout this section, we assume $\mu(\mathbb{M})=1$. Let

$$
\iota=\inf \frac{P(E)}{\mu(E)},
$$

where the infimum runs over all Caccioppoli sets $E$ such that $\mu(E) \leq 1 / 2$ (we recall that $P(E)$ denotes the perimeter of $E$ as defined in Section 2.4 ). By following the argument of Ledoux in [12] we see that $\lambda_{1} \geq i^{2} / 4$ where $\lambda_{1}$ is the first eigenvalue of $-L$. The next proposition gives a lower bound on $\iota$ (and therefore on $\lambda_{1}$ ).

Proposition 3.7. Let $E \subset \mathbb{M}$ be a Caccioppoli set. We have

$$
\mu(E)(1-\mu(E)) \leq \sqrt{\frac{2}{\rho_{1}}}\left(1+\frac{2 \kappa}{\rho_{2}}\right) P(E) .
$$

As a consequence,

$$
\iota \geq \frac{1}{2} \sqrt{\frac{\rho_{1}}{2}} \frac{1}{1+2 \kappa / \rho_{2}} .
$$

Proof. We know from the pseudo-Poincaré inequality that for $f \in C_{0}^{\infty}(\mathbb{M})$,

$$
\left\|P_{t} f-f\right\|_{1} \leq \sqrt{\frac{2}{\rho_{1}}}\left(1+\frac{2 \kappa}{\rho_{2}}\right) \sqrt{1-e^{-\frac{\rho_{1} \rho_{2}}{\rho_{2}+\kappa} t}}\|\sqrt{\Gamma(f)}\|_{1}, \quad t>0 .
$$

Suppose now that $E \subset \mathbb{M}$ is a Caccioppoli set. By Proposition 2.5 there exists a sequence $\left\{f_{n}\right\}_{n \in \mathbb{N}}$ in $C_{0}^{\infty}(\mathbb{M})$ satisfying (i) and (ii) of that proposition. Applying (3.4) to $f_{n}$, we obtain

$$
\begin{aligned}
\left\|P_{t} f_{n}-f_{n}\right\|_{1} & \leq \sqrt{\frac{2}{\rho_{1}}}\left(1+\frac{2 \kappa}{\rho_{2}}\right) \sqrt{1-e^{-\frac{\rho_{1} \rho_{2}}{\rho_{2}+\kappa} t}}\left\|\sqrt{\Gamma\left(f_{n}\right)}\right\|_{1} \\
& =\sqrt{\frac{2}{\rho_{1}}}\left(1+\frac{2 \kappa}{\rho_{2}}\right) \sqrt{1-e^{-\frac{\rho_{1} \rho_{2}}{\rho_{2}+\kappa} t}} \operatorname{Var}\left(f_{n}\right) .
\end{aligned}
$$

Letting $n \rightarrow \infty$ in this inequality, we conclude

$$
\begin{aligned}
\left\|P_{t} \mathbf{1}_{E}-\mathbf{1}_{E}\right\|_{L^{1}(\mathbb{M})} & \leq \sqrt{\frac{2}{\rho_{1}}}\left(1+\frac{2 \kappa}{\rho_{2}}\right) \sqrt{1-e^{-\frac{\rho_{1} \rho_{2}}{\rho_{2}+\kappa} t}} \operatorname{Var}\left(\mathbf{1}_{E}\right) \\
& =\sqrt{\frac{2}{\rho_{1}}}\left(1+\frac{2 \kappa}{\rho_{2}}\right) \sqrt{1-e^{-\frac{\rho_{1} \rho_{2}}{\rho_{2}+\kappa} t}} P(E) .
\end{aligned}
$$

Observe now that, using $P_{t} 1=1$, we have

$$
\begin{aligned}
\left\|P_{t} \mathbf{1}_{E}-\mathbf{1}_{E}\right\|_{L^{1}(\mathbb{M})} & \geq \int_{\mathbb{M}}\left|\mathbf{1}_{E^{c}}\right|\left|P_{t} \mathbf{1}_{E}-\mathbf{1}_{E}\right| d \mu \geq \int_{\mathbb{M}} \mathbf{1}_{E^{c}}\left(P_{t} \mathbf{1}_{E}-\mathbf{1}_{E}\right) d \mu \\
& =\int_{\mathbb{M}} \mathbf{1}_{E^{c}} P_{t} \mathbf{1}_{E} d \mu=\int_{\mathbb{M}} P_{t} \mathbf{1}_{E} d \mu-\int_{\mathbb{M}} \mathbf{1}_{E} P_{t} \mathbf{1}_{E} d \mu \\
& =\int_{\mathbb{M}} \mathbf{1}_{E} d \mu-\int_{E} P_{t} \mathbf{1}_{E} d \mu=\mu(E)-\int_{E} P_{t} \mathbf{1}_{E} d \mu
\end{aligned}
$$


On the other hand, from the semigroup property we have

$$
\int_{E} P_{t} \mathbf{1}_{E} d \mu=\int_{\mathbb{M}}\left(P_{t / 2} \mathbf{1}_{E}\right)^{2} d \mu .
$$

We thus obtain

$$
\left\|P_{t} \mathbf{1}_{E}-\mathbf{1}_{E}\right\|_{L^{1}(\mathbb{M})} \geq\left(\mu(E)-\int_{\mathbb{M}}\left(P_{t / 2} \mathbf{1}_{E}\right)^{2} d \mu\right) .
$$

In [3], it has been proved that for $x, y \in \mathbb{M}$ and $t>0$,

$$
p(x, y, t) \leq \frac{1}{\left(1-e^{-\frac{2 \rho_{1} \rho_{2} t}{3\left(\rho_{2}+\kappa\right)}}\right)^{\frac{d}{2}\left(1+3 \kappa /\left(2 \rho_{2}\right)\right)}} .
$$

This gives

$$
\begin{aligned}
\int_{\mathbb{M}}\left(P_{t / 2} \mathbf{1}_{E}\right)^{2} d \mu & \leq\left(\int_{E}\left(\int_{\mathbb{M}} p(x, y, t / 2)^{2} d \mu(y)\right)^{1 / 2} d \mu(x)\right)^{2} \\
& =\left(\int_{E} p(x, x, t)^{1 / 2} d \mu(x)\right)^{2} \leq \frac{1}{\left(1-e^{-\frac{2 \rho_{1} \rho_{2} t}{3\left(\rho_{2}+\kappa\right)}}\right)^{d\left(1+3 \kappa /\left(2 \rho_{2}\right)\right)}} \mu(E)^{2} .
\end{aligned}
$$

Combining these equations we reach the conclusion

$$
\sqrt{\frac{2}{\rho_{1}}}\left(1+\frac{2 \kappa}{\rho_{2}}\right) \sqrt{1-e^{-\frac{\rho_{1} \rho_{2}}{\rho_{2}+\kappa} t}} P(E) \geq \mu(E)-\frac{1}{\left(1-e^{-\frac{2 \rho_{1} \rho_{2} t}{3\left(\rho_{2}+\kappa\right)}}\right)^{d\left(1+3 \kappa /\left(2 \rho_{2}\right)\right)}} \mu(E)^{2} .
$$

We conclude by letting $t \rightarrow+\infty$.

\subsection{A Lichnerowicz type theorem}

A well-known theorem of Lichnerowicz asserts that on a $d$-dimensional complete Riemannian manifold whose Ricci curvature is bounded below by a nonnegative constant $\rho$, then the first eigenvalue of the Laplace-Beltrami operator is bounded below by $\rho d /(d-1)$. In this section, we provide a similar theorem for our operator L. Let us observe that in [9], Greenleaf obtained a similar result for the sub-Laplacian on a CR manifold. Recent work of Hladky [10] also gives lower bounds for the first eigenvalue of sub-Laplacians on some sub-Riemannian manifolds.

Proposition 3.8. The first nonzero eigenvalue $\lambda_{1}$ of $-L$ satisfies the estimate

$$
\lambda_{1} \geq \frac{\rho_{1} \rho_{2}}{\frac{d-1}{d} \rho_{2}+\kappa} .
$$

Proof. Let $f: \mathbb{M} \rightarrow \mathbb{R}$ be an eigenfunction corresponding to the eigenvalue $-\lambda_{1}$. From the generalized curvature dimension inequality we know that for every $\nu>0$,

$$
\Gamma_{2}(f, f)+\nu \Gamma_{2}^{Z}(f, f) \geq \frac{1}{d}(L f)^{2}+\left(\rho_{1}-\frac{\kappa}{\nu}\right) \Gamma(f, f)+\rho_{2} \Gamma^{Z}(f, f) .
$$


By integrating this inequality on the manifold $\mathbb{M}$, we obtain

$$
\begin{aligned}
\int_{\mathbb{M}} \Gamma_{2}(f, f) d \mu & +\nu \int_{\mathbb{M}} \Gamma_{2}^{Z}(f, f) d \mu \\
\geq & \frac{1}{d} \int_{\mathbb{M}}(L f)^{2} d \mu+\left(\rho_{1}-\frac{\kappa}{\nu}\right) \int_{\mathbb{M}} \Gamma(f, f) d \mu+\rho_{2} \int_{\mathbb{M}} \Gamma^{Z}(f, f) d \mu .
\end{aligned}
$$

Let us now recall that

$$
\Gamma_{2}(f, f)=\frac{1}{2}[L \Gamma(f, f)-2 \Gamma(f, L f)]
$$

and

$$
\Gamma_{2}^{Z}(f, f)=\frac{1}{2}\left[L \Gamma^{Z}(f, f)-2 \Gamma^{Z}(f, L f)\right] .
$$

Therefore, by using $L f=-\lambda_{1} f$ and integrating by parts in the above inequality, we find

$$
\left(\lambda_{1}^{2}-\frac{\lambda_{1}^{2}}{d}+\frac{\kappa \lambda_{1}}{\nu}-\rho_{1} \lambda_{1}\right) \int_{\mathbb{M}} f^{2} d \mu \geq\left(\rho_{2}-\nu \lambda_{1}\right) \int_{\mathbb{M}} \Gamma^{Z}(f, f) d \mu .
$$

By choosing $\nu=\rho_{2} / \lambda_{1}$, we obtain the inequality

$$
\lambda_{1} \geq \frac{\rho_{1} \rho_{2}}{\frac{d-1}{d} \rho_{2}+\kappa} .
$$

Remark 3.9. We note that when $\kappa=0$, which corresponds to the Riemannian case, we recover the classical theorem of Lichnerowicz.

\section{References}

[1] Baudoin, F. And Bonnefont, M.: Log-Sobolev inequalities for subelliptic operators satisfying a generalized curvature dimension inequality. J. Funct. Anal. 262 (2012), no. 6, 2646-2676.

[2] Baudoin, F., Bonnefont, M. And Garofalo, N.: A sub-Riemannian curvaturedimension inequality, volume doubling property and the Poincaré inequality. To appear in Math. Ann.

[3] Baudoin, F. and Garofalo, N.: Curvature-dimension inequalities and Ricci lower bounds for sub-Riemannian manifolds with transverse symmetries. Preprint available at: arXiv:1101.3590v4 [math.DG], 2011.

[4] Brascamp, H. J., Lieb, E. H. And Luttinger, J. M.: A general rearrangement inequality for multiple integrals. J. Functional Analysis 17 (1974), 227-237.

[5] Capogna, L., Danielli, D., Pauls, S. D. and Tyson, J. T.: An introduction to the Heisenberg group and the sub-Riemannian isoperimetric problem. Progress in Mathematics 259, Birkhäuser Verlag, Basel, 2007.

[6] Cheeger, J.: A lower bound for the smallest eigenvalue of the Laplacian. In Problems in analysis (Papers dedicated to Salomon Bochner, 1969), 195-199. Princeton Univ. Press, Princeton, N. J., 1970. 
[7] Fefferman, C. And Phong, D. H.: Subelliptic eigenvalue problems. In Conference on harmonic analysis in honor of Antoni Zygmund, Vol. I, II (Chicago, Ill., 1981), 590-606. Wadsworth Math. Ser., Wadsworth, Belmont, CA, 1983.

[8] Garofalo, N. and Nhieu, D. M.: Isoperimetric and Sobolev inequalities for Carnot-Carathéodory spaces and the existence of minimal surfaces. Comm. Pure Appl. Math. 49 (1996), no. 10, 1081-1144.

[9] Greenleaf, A.: The first eigenvalue of a sub-Laplacian on a pseudo-Hermitian manifold. Comm. Partial Differential Equations 10 (1985), no. 2, 191-217.

[10] Hladky, R.: Bounds for the first eigenvalue of the horizontal Laplacian in positively curved sub-Riemannian manifolds. Geom. Dedicata 164 (2013), 155-177.

[11] Jerison, D. And SÁnchez-Calle, A.: Subelliptic, second order differential operators. In Complex analysis, III (College Park, Md., 1985-86), 46-77. Lecture Notes in Math. 1277, Springer, Berlin, 1987.

[12] Ledoux, M.: A simple analytic proof of an inequality by P. Buser. Proc. Amer. Math. Soc. 121 (1994), no. 3, 951-959.

[13] Ledoux, M.: Isoperimetry and Gaussian analysis. In Lectures on probability theory and statistics (Saint-Flour, 1994), 165-294. Lecture Notes in Math. 1648, Springer, Berlin, 1996.

[14] Ledoux, M.: On improved Sobolev embedding theorems. Math. Res. Lett. 10 (2003), no. 5-6, 659-669.

[15] Pansu, P.: Une inégalité isopérimétrique sur le groupe de Heisenberg. C. R. Acad. Sci. Paris Sér. I Math. 295 (1982), no. 2, 127-130.

[16] Saloff-Coste, L.: Aspect of Sobolev-type inequalities. London Mathematical Society Lecture Note Series 289, Cambridge University Press, Cambridge, 2002.

[17] Wang, F. Y.: Generalized curvature condition for subelliptic diffusion processes. Preprint available at: arXiv:1202.0778v2 [math.PR], 2012.

Received March 16, 2012.

Fabrice Baudoin: Department of Mathematics, Purdue University, 150 N. University Street, West Lafayette, IN 47907-2067, USA.

E-mail: fbaudoin@math.purdue.edu

Bumsik Kim: Department of Mathematics, Purdue University, 150 N. University Street, West Lafayette, IN 47907-2067, USA.

E-mail: kim792@math.purdue.edu

The first author was supported in part by NSF Grant DMS 0907326. 